\title{
Supergravity solutions for Born-Infeld dyons
}

\author{
Donam Youm* \\ Theory Division, CERN, CH-1211, Geneva 23, Switzerland
}

(Received 25 May 1999; published 18 October 1999)

\begin{abstract}
We construct partially localized supergravity counterpart solutions to the $1 / 2$ supersymmetric nonthreshold and the 1/4 supersymmetric threshold bound state BI dyons in the D3-brane Dirac-Born-Infeld theory. Such supergravity solutions have all the parameters of the BI dyons. By applying the type-IIA-type-IIB T-duality transformations to these supergravity solutions, we obtain the supergravity counterpart solutions to $1 / 2$ and $1 / 4$ supersymmetric bions carrying electric and magnetic charges of the world volume $U(1)$ gauge field in the Dirac-Born-Infeld theory in other dimensions. [S0556-2821(99)04420-3]
\end{abstract}

PACS number(s): 11.27. $+\mathrm{d}, 04.20 . J b, 04.50 .+\mathrm{h}, 11.25 . \mathrm{Sq}$

\section{INTRODUCTION}

In the very weak string coupling limit $\left(g_{s} \rightarrow 0\right)$, the world volume theory of a D-brane is described by the Dirac-BornInfeld (DBI) action [1-5] in the flat target spacetime with vanishing forms and dilaton fields. Many of the solitons in the DBI theory, collectively called bions or BI solitons, have been studied, following the initial works in Refs. [6-8]. Bions can be regarded as the "boundary" counterparts to the "bulk" supergravity solutions in the bulk-boundary holographic duality relations [9-17]. More precisely, the intersection or the end (of one brane on the other) of an intersecting 'bulk', supergravity brane solution is interpreted in the "boundary" world volume theory as a soliton in the world volume theory [18]. Such an intersection or end is the source for the charge carried by the BI soliton [19-21]. In fact, it is observed $[6,7]$ that in the presence of a bion, which carries electric (magnetic) charge of the world volume $U(1)$ gauge field of the D3-brane DBI action, a flat D3-brane develops a transverse spike, which can be interpreted as a fundamental string (D string) ending on the D3-brane. The Coulomb energy density of such configuration is shown [7] to match the energy density of the fundamental string or the D string, supporting such an idea.

If the bulk-boundary holographic duality is correct, then all the parameters of the world volume theory or soliton have to be mapped one-to-one to the bulk theory counterparts. Generally, bions are parametrized by their charges and their locations in the world volume space. Therefore, there have to exist the corresponding localized intersecting supergravity solutions where at least one brane (interpreted as the BI soliton counterpart) is localized on the world volume of the other (where the BI soliton lives).

There have been many efforts to construct localized intersecting brane solutions [22-36]. However, it has turned out $[35,36]$ that if one works with the most general spacetime metric Ansatz for completely localized intersecting branes, the resulting equations of motion to be solved are nonlinear coupled differential equations, which do not have the general solution and are almost impossible to solve. Such equations of motion were solved only perturbatively in the case of two

\footnotetext{
*Email address: Donam.Youm@cern.ch
}

M2-branes intersecting at a point [36]. If one instead considers the restricted metric Ansatz, which is of the same form as that of the delocalized intersecting brane, the consistency of the equations of motion requires one of the branes to be delocalized on the other [22-24,33]. Even with this simplified metric Ansatz with one brane delocalized on the other, convenient closed form of solutions are generally not known, but only the solutions in terms of the infinite series of special functions can be found $[24,28]$. But when one goes to the near horizon limit of the delocalized brane, solutions can be expressed in closed form in terms of simple elementary functions [27,29-32,34]. Although such solutions are not completely localized and in some cases one has to delocalize (or compactify) some of the overall transverse directions for the purpose of localizing one brane on the other [34] (when the two constituent branes meet at the overall transverse space [37]), at this point this is the only case in which one can have explicit solutions in convenient closed forms. Perhaps, such partially localized solutions might be useful for studying the holographic relations in the restricted cases where some of directions of the world volume brane configurations are delocalized. Furthermore, such partially localized supergravity solutions have all the necessary parameters of the corresponding BI solitons, namely, the locations and the charges of bions.

In this paper, we construct the partially localized supergravity solutions which are the "bulk" counterparts to various BI solitons in the D3-brane DBI theory. Many of the partially localized supergravity solutions that are the "bulk" counterparts to the other solitons in the brane world volumes are already presented in Ref. [34]. For example, first of all for the solitons in M-brane world volume, the self-dual string [8] charged under the self-dual 3-form field strength in the M5-brane world volume corresponds to the intersecting M2and M5-branes where the M2-brane is localized at the M5brane worldvolume. The 3-brane soliton [38] in the M5brane world volume corresponds to the intersecting two M5branes where one of them is localized on the other. The neutral (with respect to the self-dual 3-form field strength) string in the M5-brane world volume [39] corresponds to the $\mathrm{M}$ wave localized in the M5-brane world volume. The zerobrane (charged with respect to the Hodge-dual of a transverse scalar) in the M2-brane world volume corresponds to the intersecting two M2-branes where one of them is local- 
ized on the other. Similarly, the partially localized intersecting branes in the ten dimensions that are presented in Ref. [34] can also be interpreted as the "bulk" counterparts to the solitons in the ten-dimensional brane worldvolumes. For example, the supergravity solution for D0-brane localized at the D4-brane is the "bulk" counterpart to the BI instanton in the world volume theory of the D4-brane [40-42], which is the double dimensional reduction of the neutral string in the M5-brane world volume. The 0-brane (2-brane) soliton on the Neveu-Schwarz 5-brane (NS5-brane) world volume corresponds to the supergravity solution for D1-brane [D3brane] localized at the NS5-brane. The 3-brane soliton on the NS5-brane world volume corresponds to the intersecting two NS5-branes where one of them is localized on the other. The solitons on the world volume of the Kaluza-Klein (KK) monopole are those on the world volume of the NS5-brane related through the type-IIA-type-IIB $T$-duality [43], e.g., the 0-brane [3-brane] soliton on the KK monopole worldvolume corresponds to the supergravity solution for the D2brane [NS5-brane] localized on the KK monopole. Furthermore, the structure of world volume supersymmetry algebra dictates the existence of such world volume solitons (charged under the central charges of the algebra) and the possible intersection rules among the spacetime branes [41].

The paper is organized as follows. In Sec. II, we survey various BI solitons in the D3-brane DBI theory. In Sec. III, we construct the corresponding supergravity solutions and their type-IIA-type-IIB $T$-duality transformed solutions.

\section{SOLITONS IN THE DIRAC-BORN-INFELD THEORY}

In this section, we summarize the previous works on solitons in the Dirac-Born-Infeld theory, which are relevant to the works in the following sections, for the purpose of completeness and fixing the notation.

\section{A. Dirac-Born-Infeld action}

In the very weak string coupling limit $\left(g_{s} \rightarrow 0\right)$, a Dpbrane moves in the flat Minkowski spacetime with trivial field configurations, i.e., constant dilaton and vanishing form fields. In this limit, a Dp-brane with the world volume coordinates $\sigma^{\mu}(\mu=0,1, \ldots, p)$ moving in a $(d+1)$ dimensional target space with the coordinates $z^{M}$ $=\left(x^{\mu}, y^{m}\right)(M=0,1, \ldots, d ; \mu=0,1, \ldots, p, m=p+1, \ldots$, $d$ ) is described by the DBI action

$$
S_{\mathrm{DBI}}=-\int d^{p+1} \sigma \sqrt{-\operatorname{det}\left(\eta_{M N} \partial_{\mu} z^{M} \partial_{\nu} z^{N}+F_{\mu \nu}\right)},
$$

where $\eta_{M N}$ is the metric for the Minkowskian target space and $F_{\mu \nu}=\partial_{\mu} A_{\nu}-\partial_{\nu} A_{\mu}$ is the field strength of the world volume $U(1)$ gauge field $A_{\mu}$.

In the "static" gauge, in which world volume coordinates $\sigma^{\mu}$ are identified with target space coordinates as $\sigma^{\mu}=x^{\mu}$, the DBI action (1) takes the following form:

$$
S_{\mathrm{DBI}}=-\int d^{p+1} \sigma \sqrt{-\operatorname{det}\left(\eta_{\mu \nu}+\partial_{\mu} y^{m} \partial_{\nu} y^{m}+F_{\mu \nu}\right)} .
$$

Since the bulk D3-brane configuration is invariant under the $S L(2, \mathbf{R})$ symmetry of the type-IIB theory, the DBI action for the D3-brane should also have the $S L(2, \mathbf{R})$ symmetry, as is proven in Refs. [44-52]. In fact, the manifestly $S L(2, \mathbf{R})$ covariant form for the D3-brane DBI action is constructed [53], which we describe in the following. The world volume $U(1)$ field strength $F$ and its dual field strength $G$, defined as $* G= \pm 2 \delta S_{\mathrm{DBI}} / \delta F$, transform as a doublet under the $S L(2, \mathbf{R}): \mathbf{F} \rightarrow \Lambda \mathbf{F}\left(\mathbf{F}=\left(\begin{array}{ll}F & G\end{array}\right)^{T}, \Lambda \in S L(2, \mathbf{R})\right)$. Furthermore, the asymptotic values of the dilaton $e^{\phi}$ and the axion $\chi$ can be identified with an $S L(2, \mathbf{R})$ doublet complex constants $U^{r}(r=1,2)$, constrained by $(i / 2) \epsilon_{r s} U^{r} \bar{U}^{s}=1$, as $U^{2} / U^{1}=\chi_{\infty}+i e^{-\phi_{\infty}}$. Here, $U$ transforms under the $S L(2, \mathbf{R})$ as $U^{T} \rightarrow U^{T} \Lambda^{-1}$. Recall that the scalars $e^{\phi}$ and $\chi$ of the type-IIB theory form the coset $S L(2, \mathbf{R}) / S O(2)$. This coset is obtained from the complex $S L(2, \mathbf{R}) \operatorname{doublet} \mathcal{U}^{r}$ by gauging the $U(1) \cong S O(2)$ which acts as $U^{r} \rightarrow e^{i \vartheta} U^{r}$. The combination $\mathcal{F}=U^{T} \mathbf{F}$ is $S L(2, \mathbf{R})$ invariant. The $S L(2, \mathbf{R})$ covariant form of the DBI action is expressed in terms of this $S L(2, \mathbf{R})$ invariant $\mathcal{F}$ and the 4-form field strength $H$ as

$$
\begin{aligned}
S_{\mathrm{D} 3}= & \int d^{4} x \lambda \sqrt{-g}\left[1+\frac{1}{2} \mathcal{F} \cdot \overline{\mathcal{F}}-\frac{1}{16}(\mathcal{F} \cdot * \mathcal{F})\right. \\
& \left.\times(\overline{\mathcal{F}} \cdot * \overline{\mathcal{F}})+\frac{1}{4} H \cdot H\right]
\end{aligned}
$$

where $\lambda$ is a Lagrange multiplier and $g$ is the determinant of the world volume metric. This action is supplemented with the following self-duality relation:

$$
\frac{i}{2}(* H) * \mathcal{F}=\mathcal{F}-\frac{1}{4}(\mathcal{F} \cdot * \mathcal{F})(\overline{\mathcal{F}} \cdot * \overline{\mathcal{F}}) * \overline{\mathcal{F}}
$$

This relation reduces the number of independent charges of $F$ and $G$ to two, which can be identified as charges whose sources are ends of fundamental string and D-string on the D3-brane.

\section{B. BI solitons}

In this subsection, we survey various solitons in the DBI theory whose corresponding and duality related supergravity solutions we will construct in the following section.

By applying the $S L(2, \mathbf{R})$ transformation, one can bring arbitrary constants $U^{r}$ to $U^{1}=1$ and $U^{2}=i$, corresponding to the asymptotic values $e^{\phi_{\infty}}=1$ and $\chi_{\infty}=0$ for the type-IIB theory scalars. Note, this choice of the constants $U^{i}$ is preserved by the $U(1)$ subgroup transformation discussed in the previous subsection, just like the $S O(2) \subset S L(2, \mathbf{R})$ transformation leaves the scalar asymptotic values $e^{\phi_{\infty}}=1$ and $\chi_{\infty}$ 
$=0$ intact. With this choice, the complex $U(1)$ field strength takes the form $\mathcal{F}=F+i G$.

In this case, a general solution, which preserves $1 / 4$ of the world volume supersymmetry, has the following form $[6-8,42,54,55]$ :

$$
\begin{aligned}
& \mathbf{D}=\nabla \sum_{i=1}^{N_{q}} \frac{q_{i}}{\left|\mathbf{r}-\mathbf{x}_{i}\right|}, \quad X=\sum_{i=1}^{N_{q}} \frac{q_{i}}{\left|\mathbf{r}-\mathbf{x}_{i}\right|}, \\
& \mathbf{B}=\nabla \sum_{i=1}^{N_{p}} \frac{p_{i}}{\left|\mathbf{r}-\mathbf{y}_{i}\right|}, \quad Y=\sum_{i=1}^{N_{p}} \frac{p_{i}}{\left|\mathbf{r}-\mathbf{y}_{i}\right|},
\end{aligned}
$$

where $X$ and $Y$ are scalars describing target space coordinates perpendicular to the world volume, and $\mathbf{r}=\left(\sigma^{1}, \sigma^{2}, \sigma^{3}\right)$ is the spatial world volume coordinates. Here, $\mathbf{D}$ is the canonical momentum conjugate to $\mathbf{A}=\left(A_{i}\right)$, i.e., the electric induction, and $\mathbf{B}$ is the magnetic induction $\left(B_{i}=\frac{1}{2} \epsilon_{i j k} F_{j k}\right)$. The solution describes electric and magnetic bions located at different points in the world volume. The bulk interpretation of this solution is that fundamental strings stretching in the $X$ direction end on the D3-brane at $\mathbf{x}_{i}$ and D strings in the Y direction end at $\mathbf{y}_{i}$. The parameters $q_{i}$ and $p_{i}$ are, respectively, related to charges of the fundamental strings and $D$ strings.

A special case of this general solution which is also Bogomol'nyi-Prasad-Sommerfield (BPS) is the case when $N:=N_{q}=N_{p}$ and $\mathbf{x}_{i}=\mathbf{y}_{i}$. Such a solution describes $N$ BI dyons with charges $\left(q_{i}, p_{i}\right)$ located at $\mathbf{x}_{i}$. The bulk interpretation is $N$ dyonic strings, each with the NS-NS and the Raymond-Raymond (R-R) two-form potential charges related to $\left(q_{i}, p_{i}\right)$, ending at the points $\mathbf{r}=\mathbf{x}_{i}$ in the D3-brane world volume and stretched in the directions $q_{i} \hat{\mathbf{e}}_{X}+p_{i} \hat{\mathbf{e}}_{Y}$. Here, $\hat{\mathbf{e}}_{X}$ and $\hat{\mathbf{e}}_{Y}$ are, respectively, the unit vectors in the $X$ and $Y$ directions. In this case, $1 / 4$ of spacetime supersymmetry is preserved. The particular case in which both electric and magnetic bions have two centers is interpreted as the string junction [56]. This can be seen by studying the solution in the three asymptotic regions near the two centers ( $\mathbf{r}$ $\rightarrow \mathbf{x}_{1}$ and $\mathbf{r} \rightarrow \mathbf{x}_{2}$ ) of the bions and far way from the bions $(|\mathbf{r}| \rightarrow \infty)$. The angles between strings in the string junction are determined by the charges $q_{1}, q_{2}, p_{1}$, and $p_{2}$ of the bions, and are consistent with charge conservation and tension balance, necessary for the existence of the static BPS string junction configuration [57-59].

The BI soliton that corresponds to the 1/2 BPS type-IIB dyonic string of Schwarz [45] can be similarly constructed [55]. One starts with the special case of the general solution (5) in which $p_{i}=0$ and only one of $q_{i}$ is nonzero, say, $q_{1}$ $=q \Delta_{(m, n)}^{1 / 2}$ and $\mathbf{x}_{1}=\mathbf{r}_{0}$. Here, we redefined the charge with an arbitrary multiplicative constant $\Delta_{(m, n)}$ for the purpose of charge quantization after the $S L(2, \mathbf{R})$ transformation. This corresponds to a fundamental string ending on D3-brane. In order to get the BI dyon with electric and magnetic charges $(m q, n q)$ and an arbitrary scalar asymptotic value $\tau_{\infty}=\chi_{\infty}$ $+i e^{-\phi_{\infty}}$, one applies the $S L(2, \mathbf{R})$ transformation with the following matrix:

$$
\Gamma=\left(\begin{array}{cc}
e^{-\phi_{\infty} / 2} & \chi_{\infty} e^{\phi_{\infty} / 2} \\
0 & e^{\phi_{\infty} / 2}
\end{array}\right)\left(\begin{array}{cc}
\cos \alpha & -\sin \alpha \\
\sin \alpha & \cos \alpha
\end{array}\right)
$$

with the $S O(2)$ rotation angle $\alpha$ constrained to satisfy

$$
\begin{aligned}
& \cos \alpha=e^{\phi_{\infty} / 2}\left(m-n \chi_{\infty}\right) \Delta_{(m, n)}^{-1 / 2}, \\
& \sin \alpha=e^{-\phi_{\infty} / 2} n \Delta_{(m, n)}^{-1 / 2} ; \quad m, n \in \mathbf{Z},
\end{aligned}
$$

which fix the expression for $\Delta_{(m, n)}$ as

$$
\Delta_{(m, n)}=n^{2} e^{-\phi_{\infty}}+\left(n \chi_{\infty}-m\right)^{2} e^{\phi_{\infty}} .
$$

The resulting dyonic solution has the following form:

$$
\mathbf{D}=\nabla \frac{m q}{\left|\mathbf{r}-\mathbf{r}_{0}\right|}, \quad \mathbf{B}=\nabla \frac{n q}{\left|\mathbf{r}-\mathbf{r}_{0}\right|}, \quad X=\frac{\Delta_{(m, n)}^{1 / 2} q}{\left|\mathbf{r}-\mathbf{r}_{0}\right|}
$$

This solution is interpreted as the Schwarz dyonic string [45] with the charges related to $(m q, n q)$ and stretching in the $X$ direction ending on D3-brane.

\section{SUPERGRAVITY SOLUTIONS}

In this section, we construct the partially localized supergravity solution counterparts to the BI solitons in the D3brane world volume discussed in the previous section.

\section{A. Type-IIB supergravity and the $S L(2, \mathrm{R})$ symmetry}

In this subsection, we summarize the bosonic effective supergravity action for the type-IIB superstring and its $S L(2, \mathbf{R})$ symmetry for the completeness and for the purpose of fixing the notation.

The bosonic part of the low energy effective supergravity theory for the type-IIB string theory is described by the massless bosonic string modes. These are the graviton $G_{M N}^{\mathrm{str}}$, the 2-form potential $B_{M N}^{(1)}$ and the dilaton $\phi$ in the NS-NS sector, and the axionic scalar $\chi$, the 2-form potential $B_{M N}^{(2)}$ and the 4-form potential $D_{M N P O}$ in the R-R sector. In the string frame, the bosonic part of the effective supergravity action has the following form [60]:

$$
\begin{aligned}
S_{\mathrm{IIB}}^{\mathrm{str}}= & \frac{1}{2} \int d^{10} x \sqrt{-G^{\mathrm{str}}}\left[e^{-2 \phi}\left\{-\mathcal{R}^{\mathrm{str}}+4(\partial \phi)^{2}-\frac{3}{4}\left(H^{(1)}\right)^{2}\right\}\right. \\
& -\frac{1}{2}(\partial \chi)^{2}-\frac{3}{4}\left(H^{(2)}-\chi H^{(1)}\right)^{2}-\frac{5}{6} F^{2} \\
& \left.-\frac{1}{96 \sqrt{-G^{\mathrm{str}}}} \epsilon^{i j} \epsilon D H^{(i)} H^{(j)}\right],
\end{aligned}
$$


where $H^{(i)}=\partial B^{(i)} \quad(i=1,2)$ and $F=\partial D+\frac{3}{4} \epsilon^{i j} B^{(i)} B^{(j)}$ are, respectively, the field strengths of the potentials $B^{(i)}$ and $D$.

The $S L(2, \mathbf{R})$ symmetry $[61,62]$ of the type-IIB theory is manifest explicitly in the effective action in the Einstein frame. By applying the Weyl-scaling transformation of spacetime metric, $G_{M N}=e^{-\phi / 2} G_{M N}^{\mathrm{str}}$, one can bring the action (10) to the following Einstein frame form [60]:

$$
\begin{aligned}
S_{\mathrm{IIB}}^{E}= & \frac{1}{2} \int d^{10} x \sqrt{-G}\left[-\mathcal{R}+\frac{1}{4} \operatorname{Tr}\left(\partial_{M} \mathcal{M} \partial^{M} \mathcal{M}\right)\right. \\
& \left.-\frac{3}{4} H^{(i)} \mathcal{M}_{i j} H^{(j)}-\frac{5}{6} F^{2}-\frac{1}{96 \sqrt{-G}} \epsilon^{i j} \epsilon D H^{(i)} H^{(j)}\right],
\end{aligned}
$$

where an $S L(2, \mathbf{R})$ invariant matrix $\mathcal{M}$ formed by the scalar $\lambda \equiv \chi+i e^{-\phi}$ has the following form:

$$
\mathcal{M}=e^{\phi}\left(\begin{array}{cc}
|\lambda|^{2} & \chi \\
\chi & 1
\end{array}\right)
$$

The Einstein frame action (11) is invariant under the following $S L(2, \mathbf{R})$ transformation:

$$
\left(\begin{array}{l}
B^{(1)} \\
B^{(2)}
\end{array}\right) \rightarrow\left(\Lambda^{T}\right)^{-1}\left(\begin{array}{l}
B^{(1)} \\
B^{(2)}
\end{array}\right), \quad \mathcal{M} \rightarrow \Lambda \mathcal{M} \Lambda^{T} ; \quad \Lambda \in S L(2, \mathbf{R}),
$$

with the metric $G_{M N}$ and the 4 -form potential $D$ remaining intact.

\section{B. Supergravity solution for the nonthreshold bound state BI dyon}

To construct the supergravity counterpart solution to the 1/2 supersymmetric BI dyon solution in Eq. (9), we start with the following partially localized supergravity solution for the fundamental string ending on ${ }^{1}$ D3-brane [34]:

$$
\begin{aligned}
G_{M N}^{\mathrm{str}} d x^{M} d x^{N}= & -H_{F}^{-1} H_{3}^{-1 / 2} d t^{2}+H_{3}^{-1 / 2}\left(d x_{1}^{2}+d x_{2}^{2}+d x_{3}^{2}\right) \\
& +H_{F}^{-1} H_{3}^{1 / 2} d y^{2}+H_{3}^{1 / 2}\left(d z_{1}^{2}+\cdots+d z_{5}^{2}\right), \\
e^{\phi}= & H_{F}^{-1 / 2}, \quad B_{t y}^{(1)}=H_{F}^{-1}, \quad D_{t x_{1} x_{2} x_{3}}=H_{3}^{-1},
\end{aligned}
$$

\footnotetext{
${ }^{1}$ Strictly speaking, the following solution corresponds to the fundamental string "piercing through" D3-brane, rather than ending on D3-brane. However, at this point, this solution is the closest supergravity solution that we have.
}

where the harmonic functions $H_{F}$ and $H_{3}$, respectively, associated with the fundamental string and the D3-brane are given $b^{2}$

$$
H_{F}=1+\frac{Q_{F}}{\left[\left|\vec{x}-\vec{x}_{0}\right|^{2}+4 Q_{3}\left|\vec{z}-\vec{z}_{0}\right|\right]^{3}}, \quad H_{3}=\frac{Q_{3}}{\left|\vec{z}-\vec{z}_{0}\right|} .
$$

Note, in the above two overall transverse directions are delocalized (i.e., $\vec{z}$ and $\vec{z}_{0}$ in the above harmonic functions are 3 -vectors) so that the fundamental string can be localized along the world volume directions of the D3-brane.

In the Einstein-frame, in which the $S L(2, \mathbf{R})$ symmetry is explicitly manifest in the type-IIB supergravity action, the spacetime metric takes the following form:

$$
\begin{aligned}
G_{M N} d x^{M} d x^{N}= & e^{-\phi / 2} G_{M N}^{\mathrm{str}} d x^{M} d x^{N} \\
= & -H_{F}^{-3 / 4} H_{3}^{-1 / 2} d t^{2}+H_{F}^{1 / 4} H_{3}^{-1 / 2}\left(d x_{1}^{2}+d x_{2}^{2}\right. \\
& \left.+d x_{3}^{2}\right)+H_{F}^{-3 / 4} H_{3}^{1 / 2} d y^{2}+H_{F}^{1 / 4} H_{3}^{1 / 2}\left(d z_{1}^{2}+\cdots\right. \\
& \left.+d z_{5}^{2}\right) .
\end{aligned}
$$

In order to construct the supergravity solution corresponding to the $1 / 2$ BPS BI dyon, we apply the $S L(2, \mathbf{R}) S$-duality transformation of the type-IIB supergravity. We follow the prescription discussed in Ref. [45]. Before one applies the $S L(2, \mathbf{R})$ transformation (13) to the supergravity solution (14) for the fundamental string ending on the D3-brane, one has to first modify the charge $Q_{F}$ of the fundamental string by multiplying it with an arbitrary constant, i.e., $Q_{F}$ $\rightarrow Q_{\left(q_{1}, q_{2}\right)} \equiv \Delta_{\left(q_{1}, q_{2}\right)}^{1 / 2} Q_{F}$, for the purpose of recovering the charge quantization condition [63-69] after the $S L(2, \mathbf{R})$ transformation.

\footnotetext{
${ }^{2}$ The coupled differential equations that these harmonic functions satisfy have the $\delta$-function source terms, which correspond to the (microscopic brane) sources to the brane charges. Namely, strictly speaking of the coupled differential equations, given in Ref. [34], that the harmonic functions $H_{F}$ and $H_{3}$ satisfy have to be of the following forms:

$$
\begin{gathered}
\partial_{z}^{2} H_{F}+H_{3} \partial_{\vec{x}}^{2} H_{F}=q_{F} \delta\left(\vec{x}-\vec{x}_{0}\right) \delta\left(\vec{z}-\vec{z}_{0}\right), \\
\partial_{z}^{2} H_{3}=\frac{q_{3}}{V_{y}} \delta\left(\vec{z}-\vec{z}_{0}\right),
\end{gathered}
$$

where $q_{3}$ and $q_{F}$ are, respectively, charges of the fundamental string and the D3-brane and $V_{y}$ is the volume of the delocalized $y$ space. Similarly, the differential equations in Eq. (33) have to be modified by the $\delta$-function source terms. Although the partially localized supergravity solutions presented in this paper are valid only in the near-horizon region of one of the constituents, one can still define charges of the constituent branes through Gauss' law by doing volume integrals over the spaces that contain the $\delta$-function singularities of the brane charge sources.
} 
We choose the following $S L(2, \mathbf{R})$ matrix for the transformations:

$$
\begin{aligned}
\Lambda & =\Lambda_{1} \Lambda_{2}=\left(\begin{array}{cc}
e^{-\phi_{\infty} / 2} & \chi_{\infty} e^{\phi_{\infty} / 2} \\
0 & e^{\phi_{\infty} / 2}
\end{array}\right)\left(\begin{array}{cc}
\cos \alpha & -\sin \alpha \\
\sin \alpha & \cos \alpha
\end{array}\right) \\
& =\left(\begin{array}{cc}
e^{-\phi_{\infty}} \cos \alpha+\chi_{\infty} \sin \alpha & -e^{-\phi_{\infty}} \sin \alpha+\chi_{\infty} \cos \alpha \\
\sin \alpha & \cos \alpha
\end{array}\right) e^{\phi_{\infty} / 2} .
\end{aligned}
$$

The transformation by an $S O(2)$ matrix $\Lambda_{2}$, which is the most general $S L(2, \mathbf{R})$ matrix that preserves the asymptotic value $\mathcal{M}_{\infty}=I$ (or $\lambda_{\infty}=i$ ) of the scalar matrix $\mathcal{M}$, on the solution (14) results in the supergravity solution for a nonthreshold bound state of the fundamental string and the $D$ string ending on the D3 brane with the asymptotic value $\mathcal{M}_{\infty}=I$ of the scalar $\mathcal{M}$. The subsequent transformation on the transformed solution by the matrix $\Lambda_{1}$ leads to the solution with an arbitrary asymptotic value $\mathcal{M}_{\infty}$ (or $\lambda_{\infty}=\chi_{\infty}$ $\left.+i e^{-\phi_{\infty}}\right)$ for the scalar fields.

By demanding that the $S L(2, \mathbf{R})$ transformed charges $Q^{(1)}$ and $Q^{(2)}$ for the fundamental string and the $D$ string to be quantized, i.e.,

$$
\begin{aligned}
& Q^{(1)}=\left(e^{-\phi_{\infty} / 2} \cos \alpha+\chi_{\infty} e^{\phi_{\infty} / 2} \sin \alpha\right) \Delta_{\left(q_{1}, q_{2}\right)}^{1 / 2} Q_{F}=: q_{1} Q_{F}, \\
& Q^{(2)}=e^{\phi_{\infty} / 2} \sin \alpha \Delta_{\left(q_{1}, q_{2}\right)}^{1 / 2} Q_{F}=: q_{2} Q_{F},
\end{aligned}
$$

for any integers $q_{1}$ and $q_{2}$, one fixes the $S O(2)$ rotation angle $\alpha$ to be

$$
\begin{aligned}
& \left.\begin{array}{l}
\cos \alpha=e^{\phi_{\infty} / 2}\left(q_{1}-q_{2} \chi_{\infty}\right) \Delta_{\left(q_{1}, q_{2}\right)}^{-1 / 2} \\
\sin \alpha=e^{-\phi_{\infty} / 2} q_{2} \Delta_{\left(q_{1}, q_{2}\right)}^{-1 / 2}
\end{array}\right\} \\
& \Leftrightarrow e^{i \alpha}=e^{\phi_{\infty} / 2}\left(q_{1}-q_{2} \bar{\lambda}_{\infty}\right) \Delta_{\left(q_{1}, q_{2}\right)}^{-1 / 2} ; \quad q_{1}, q_{2} \in \mathbf{Z} .
\end{aligned}
$$

From this, one determines the expression for the arbitrary constant $\Delta_{\left(q_{1}, q_{2}\right)}$ to be

$$
\begin{aligned}
& \Delta_{\left(q_{1}, q_{2}\right)} \equiv e^{\phi_{\infty}}\left(q_{1}-q_{2} \chi_{\infty}\right)^{2}+e^{-\phi_{\infty}} q_{2}^{2} \\
& =\left(\begin{array}{ll}
q_{1} & q_{2}
\end{array}\right) \mathcal{M}_{\infty}^{-1}\left(\begin{array}{l}
q_{1} \\
q_{2}
\end{array}\right) .
\end{aligned}
$$

The $\quad S L(2, \mathbf{R}) \quad$ transformed charges $\left(Q^{(1)}, Q^{(2)}\right)$ $=\left(q_{1} Q_{F}, q_{2} Q_{F}\right)$ of the fundamental string and the $D$ string satisfy the charge quantization condition.

The final form of the $S L(2, \mathbf{R})$ transformed solution in the Einstein frame describing the nonthreshold bound state of the fundamental string and the $D$ string ending on the D3brane with arbitrary asymptotic values for the scalars is as follows:

$$
\begin{gathered}
G_{M N} d x^{M} d x^{N}=-H_{\left(q_{1}, q_{2}\right)}^{-3 / 4} H_{3}^{-1 / 2} d t^{2}+H_{\left(q_{1}, q_{2}\right)}^{1 / 4} H_{3}^{-1 / 2}\left(d x_{1}^{2}\right. \\
\left.+d x_{2}^{2}+d x_{3}^{2}\right)+H_{\left(q_{1}, q_{2}\right)}^{-3 / 4} H_{3}^{1 / 2} d y^{2} \\
+H_{\left(q_{1}, q_{2}\right)}^{1 / 4} H_{3}^{1 / 2}\left(d z_{1}^{2}+\cdots+d z_{5}^{2}\right), \\
\lambda=\frac{q_{1} \chi_{\infty}-q_{2}\left|\lambda_{\infty}\right|^{2}+i q_{1} e^{-\phi_{\infty}} H_{\left(q_{1}, q_{2}\right)}^{1 / 2}}{q_{1}-q_{2} \chi_{\infty}+i q_{2} e^{-\phi_{\infty}} H_{\left(q_{1}, q_{2}\right)}^{1 / 2}}, \\
\left(\begin{array}{l}
B_{t y}^{(1)} \\
B_{t y}^{(2)}
\end{array}\right)=\mathcal{M}_{\infty}^{-1}\left(\begin{array}{l}
q_{1} \\
q_{2}
\end{array}\right) \Delta_{\left(q_{1}, q_{2}\right)}^{-1 / 2} H_{\left(q_{1}, q_{2}\right)}^{-1}, \quad D_{t x_{1} x_{2} x_{3}}=H_{3}^{-1} .
\end{gathered}
$$

From the above expression for $\lambda$, one has the following forms for the axion $\chi$ and the dilaton $e^{\phi}$ :

$$
\begin{gathered}
\chi=\frac{e^{\phi_{\infty}} \chi_{\infty} \Delta_{\left(q_{1}, q_{2}\right)}+q_{1} q_{2}\left(H_{\left(q_{1}, q_{2}\right)}-1\right)}{q_{2}^{2} H_{\left(q_{1}, q_{2}\right)}+e^{2 \phi_{\infty}}\left(q_{1}-q_{2} \chi_{\infty}\right)^{2}}, \\
e^{-\phi}=\frac{\Delta_{\left(q_{1}, q_{2}\right)} H_{\left(q_{1}, q_{2}\right)}^{1 / 2}}{q_{2}^{2} H_{\left(q_{1}, q_{2}\right)}+e^{2 \phi_{\infty}}\left(q_{1}-q_{2} \chi_{\infty}\right)^{2}} \\
=: e^{-\phi_{\infty} \bar{H}_{\left(q_{1}, q_{2}\right)}^{-1} H_{\left(q_{1}, q_{2}\right)}^{1 / 2},}
\end{gathered}
$$

where

$$
\bar{H}_{\left(q_{1}, q_{2}\right)}=\frac{e^{-\phi_{\infty}} q_{2}^{2} H_{\left(q_{1}, q_{2}\right)}+e^{\phi_{\infty}}\left(q_{1}-q_{2} \chi_{\infty}\right)^{2}}{\Delta_{\left(q_{1}, q_{2}\right)}} .
$$

Here, the harmonic functions, which describe the nonthreshold bound state of the fundamental string and the $D$ string localized on the world volume of the D3-brane, are given by

$$
\begin{aligned}
H_{\left(q_{1}, q_{2}\right)} & =1+\frac{Q_{\left(q_{1}, q_{2}\right)}}{\left[\left|\vec{x}-\vec{x}_{0}\right|^{2}+4 Q_{3}\left|\vec{z}-\vec{z}_{0}\right|\right]^{3}}, \\
\bar{H}_{\left(q_{1}, q_{2}\right)} & =1+\frac{e^{-\phi_{\infty}} q_{2}^{2} \Delta_{\left(q_{1}, q_{2}\right)}^{-1} Q_{\left(q_{1}, q_{2}\right)}}{\left[\left|\vec{x}-\vec{x}_{0}\right|^{2}+4 Q_{3}\left|\vec{z}-\vec{z}_{0}\right|\right]^{3}}, \\
H_{3} & =\frac{Q_{3}}{\left|\vec{z}-\vec{z}_{0}\right|},
\end{aligned}
$$

where $Q_{\left(q_{1}, q_{2}\right)}=\Delta_{\left(q_{1}, q_{2}\right)}^{1 / 2} Q_{F}$.

Note, this solution has all the parameters of the $1 / 2$ BPS BI dyon, namely, $\vec{x}_{0}$ that is related to the location of the BI dyon in the D3-brane world volume and $\left(q_{1}, q_{2}\right)$ that are related to the electric and the magnetic charges of the $\mathrm{BI}$ dyon. Note also that the parameter $q \Delta_{(m, n)}$ in the expression for $X$ in Eq. (9) is related to $Q_{\left(q_{1}, q_{2}\right)}$ in the above harmonic function $H_{\left(q_{1}, q_{2}\right)}$ for the $\left(q_{1} Q_{F}, q_{2} Q_{F}\right)$ string. This is related to the fact that the magnitude of the scalar charge [of the scalar $X$ in Eq. (9)] can be identified with the tension of the attached string, i.e., the above $\left(q_{1} Q_{F}, q_{2} Q_{F}\right)$ string. The di- 
rection along which the $\left(q_{1} Q_{F}, q_{2} Q_{F}\right)$ string stretches is the direction associated with the world volume scalar $X$.

By applying the type-IIA-type-IIB $T$-duality transformations on the solution (22) along the overall transverse directions $z_{i}$, one obtains partially localized supergravity solutions that correspond to a new class of nonthreshold bound state BI solitons. Before one applies the $T$-duality transformations, one has to first delocalize the solution along those directions, i.e., one has to compactify the $T$-dualized directions on circles. Note, however, that two of the overall transverse directions of the solution (22) are already delocalized for the purpose of localizing the string on the world volume of the D3-brane. The resulting supergravity solution has the following form:

$$
\begin{aligned}
G_{M N} d x^{M} d x^{N}= & e^{(n+2) \phi_{\infty} / 8} \bar{H}_{1+n}^{(n+2) / 8} H_{1}^{1 / 4} H_{3+n}^{(n-4) / 8}\left[-H_{1}^{-1} d t^{2}\right. \\
& +d x_{1}^{2}+d x_{2}^{2}+d x_{3}^{2}+e^{-\phi_{\infty}} \bar{H}_{1+n}^{-1}\left(d u_{1}^{2}+\cdots\right. \\
& \left.+d u_{n}^{2}\right)+H_{1}^{-1} H_{3+n} d y^{2}+H_{3+n}\left(d z_{1}^{2}+\cdots\right. \\
& \left.\left.+d z_{4-n}^{2}\right)\right], \\
e^{\phi}= & e^{(2-n) \phi_{\infty} / 4} \bar{H}_{1+n}^{(2-n) / 4} H_{1}^{-1 / 2} H_{3+n}^{-n / 4},
\end{aligned}
$$

where in the case of $n=1,2$ the harmonic functions are given by

$$
\begin{aligned}
H_{1} & =1+\frac{Q_{\left(q_{1}, q_{2}\right)}}{\left[\left|\vec{x}-\vec{x}_{0}\right|^{2}+4 Q_{3}\left|\vec{z}-\vec{z}_{0}\right|\right]^{3}}, \\
\bar{H}_{1+n} & =1+\frac{e^{-\phi_{\infty} q_{2}^{2} \Delta_{\left(q_{1}, q_{2}\right)}^{-1} Q_{\left(q_{1}, q_{2}\right)}}}{\left[\left|\vec{x}-\vec{x}_{0}\right|^{2}+4 Q_{3}\left|\vec{z}-\vec{z}_{0}\right|\right]^{3}}, \\
H_{3+n} & =\frac{Q_{3}}{\left|\vec{z}-\vec{z}_{0}\right|} .
\end{aligned}
$$

The solution describes the nonthreshold bound state of the fundamental string (with the longitudinal coordinate $y$ and the associated harmonic function $H_{1}$ ) and the $\mathrm{D}(1+n)$-brane [with the longitudinal coordinates $\left(y, u_{1}, \ldots, u_{n}\right)$ and the associated harmonic function $\bar{H}_{1+n}$ ending on the $\mathrm{D}(3+n)$-brane with the longitudinal coordinates $\left(x_{1}, x_{2}, x_{3}, u_{1}, \ldots, u_{n}\right)$ and the associated harmonic function $\mathrm{H}_{3+n}$ ].

From this solution, one can see the existence of the $1 / 2$ BPS nonthreshold bound state of an electric bion and a magnetic bion under the world volume $U(1)$ gauge field of the $(3+n)$-dimensional DBI theory. Such magnetic bion is related to the self-dual string [8] in the world volume of the M5-brane through the dimensional reduction (along the world volume direction transverse to the self-dual string) and the type-IIA-type-IIB $T$-dualities (in the transverse directions). Also, the electric bion has the same origin in the M5brane world volume: for this case, the longitudinal direction of the self-dual string is compactified. One can think of such nonthreshold bound states of the electric and magnetic bions in the $\mathrm{D}(3+n)$-brane world volume as being originated from the self-dual string soliton in the M5-brane world volume wrapped around a "tilted" circle (at angle with respect to the longitudinal and a transverse direction) [70,8], followed by the type-IIA-type-IIB $T$-duality transformations.

In the string frame, the above solution (26) takes more recognizable form. After redefining the scalar asymptotic values such that the resulting spacetime metric in the string frame is asymptotically flat and then applying the Weylrescale transformation of the metric $G_{M N}^{\mathrm{str}}=e^{\phi / 2} G_{M N}$, one obtains the following string frame form of the spacetime metric:

$$
\begin{aligned}
G_{M N}^{\mathrm{str}} d x^{M} d x^{N}= & -H_{1}^{-1} \bar{H}_{1+n}^{1 / 2} H_{3+n}^{-1 / 2} d t^{2}+\bar{H}_{1+n}^{1 / 2} H_{3+n}^{-1 / 2} \\
& \times\left(d x_{1}^{2}+d x_{2}^{2}+d x_{3}^{2}\right)+\bar{H}_{1+n}^{-1 / 2} H_{3+n}^{-1 / 2} \\
& \times\left(d u_{1}^{2}+\cdots+d u_{n}^{2}\right)+H_{1}^{-1} \bar{H}_{1+n}^{1 / 2} H_{3+n}^{1 / 2} d y^{2} \\
& +\bar{H}_{1+n}^{1 / 2} H_{3+n}^{1 / 2}\left(d z_{1}^{2}+\cdots+d z_{4-n}^{2}\right)
\end{aligned}
$$

\section{Supergravity solution for the threshold bound state BI dyon}

The "bulk" spacetime configuration counterpart to the threshold bound state of electric bions and magnetic bions is the orthogonal fundamental and $D$ strings ending on D3brane with the following configuration:

$$
\begin{array}{ccccccccccc}
D 3: & 1 & 2 & 3 & - & - & - & - & - & - \\
D 1: & - & - & - & 4 & - & - & - & - & - \\
F 1: & - & - & - & - & 5 & - & - & - & -
\end{array}
$$

In the string frame, such configuration is described by the following supergravity solution, which is the "bulk" counterpart to the general BI dyon in Eq. (5):

$$
\begin{gathered}
G_{M N}^{\mathrm{str}} d x^{M} d x^{N}=-H_{F}^{-1} H_{1}^{-1 / 2} H_{3}^{-1 / 2} d t^{2}+H_{1}^{1 / 2} H_{3}^{-1 / 2}\left(d x_{1}^{2}+d x_{2}^{2}\right. \\
\left.+d x_{3}^{2}\right)+H_{1}^{-1 / 2} H_{3}^{1 / 2} d u^{2}+H_{F}^{-1} H_{1}^{1 / 2} H_{3}^{1 / 2} d y^{2} \\
+H_{1}^{1 / 2} H_{3}^{1 / 2}\left(d z_{1}^{2}+\cdots+d z_{4}^{2}\right) \\
e^{\phi=} H_{F}^{-1 / 2} H_{1}^{1 / 2}, \quad B_{t y}^{(1)}=H_{F}^{-1}, \quad B_{t u}^{(2)}=H_{1}^{-1} \\
D_{t x_{1} x_{2} x_{3}}=H_{3}^{-1},
\end{gathered}
$$

where the harmonic functions satisfy the following coupled differential equations:

$$
\begin{aligned}
& \partial_{\vec{z}}^{2} H_{F}+H_{3} \partial_{\vec{x}}^{2} H_{F}+H_{1} \partial_{u}^{2} H_{F}=0, \\
& \partial_{\vec{z}}^{2} H_{1}+H_{3} \partial_{x}^{2} H_{1}+H_{F} \partial_{y}^{2} H_{3}=0, \\
& \partial_{\vec{z}}^{2} H_{3}+H_{1} \partial_{u}^{2} H_{3}+H_{F} \partial_{y}^{2} H_{3}=0,
\end{aligned}
$$

along with the constraints

$$
\partial_{u} H_{F} \partial_{y} H_{p}=0, \quad \partial_{y} H_{3} \partial_{x}^{-} H_{F}=0, \quad \partial_{u} H_{3} \partial_{\vec{x}}^{\vec{x}} H_{1}=0 .
$$


The constraints (32) can be satisfied when the solution (30) is delocalized along $y$ and $u$ directions. With this choice, the fundamental strings and the $D$ strings become completely localized at the D3-brane. In this case, the coupled differential equations (31) satisfied by the harmonic functions reduce to

$$
\partial_{z}^{2} H_{F}+H_{3} \partial_{\vec{x}}^{2} H_{F}=0, \quad \partial_{\vec{z}}^{2} H_{1}+H_{3} \partial_{\vec{x}}^{2} H_{1}=0, \quad \partial_{\vec{z}}^{2} H_{3}=0 .
$$

One can solve these coupled differential equations by following the procedure discussed in Ref. [34]. Since the dimensionality of the overall transverse space is 4 , one has to delocalize one of the overall transverse directions in order for the fundamental string and the $D$ string to be localized along the world volume directions of the D3-brane. The expressions for the harmonic functions have the following forms:

$$
\begin{aligned}
& H_{F}=1+\sum_{i=1}^{N_{F}} \frac{Q_{F i}}{\left[\left|\vec{x}-\vec{x}_{F 1 i}\right|^{2}+4 Q_{3}\left|\vec{z}-\vec{z}_{0}\right|\right]^{3 / 2}}, \\
& H_{1}=1+\sum_{i=1}^{N_{D}} \frac{Q_{D i}}{\left[\left|\vec{x}-\vec{x}_{D 1 i}\right|^{2}+4 Q_{3}\left|\vec{z}-\vec{z}_{0}\right|\right]^{3 / 2}}, \\
& H_{3}=\frac{Q_{3}}{\left|\vec{z}-\vec{z}_{0}\right|} .
\end{aligned}
$$

Note, these "localized" harmonic functions contain all the parameters of the electric bions and the magnetic bions in the world volume of the D3-brane. Namely, the electric and magnetic charges $q_{i}$ and $p_{i}$ of the bions (5) are related to the charges $Q_{F i}$ and $Q_{D i}$ of the fundamental strings and the $D$ strings, and the locations $\mathbf{x}_{i}$ and $\mathbf{y}_{i}$ of the electric bions and the magnetic bions are related to the locations $\vec{x}_{F 1 i}$ and $\vec{x}_{D 1 i}$ of the fundamental strings and the $D$ strings in the D3-brane world volume directions.

As in the case of the threshold bound state BI dyon solution (5), when $N_{F}=N_{D}$ and $\vec{x}_{F 1 i}=\vec{x}_{D 1 i}$ the solution (30) describes the threshold bound state of dyonic strings with the NS-NS and R-R two-form charges $\left(Q_{F i}, Q_{D i}\right)$ stretched in the $Q_{F i} \hat{\mathbf{e}}_{y}+Q_{D i} \hat{\mathbf{e}}_{u}$ directions. Here, $\hat{\mathbf{e}}_{y}$ and $\hat{\mathbf{e}}_{u}$ are, respectively, the unit vectors in the $y$ and $u$ directions. Since these dyons are at angle with respect to the $y$ and $u$ coordinates, there has to be a nonzero off-diagonal term $G_{u y}^{\text {str }}$ indicating rotation of the dyonic strings in the $u y$-plane. But the above supergravity solution lacks such a term, because the solution is delocalized along those directions. Such an off-diagonal term is expected to appear in the fully localized solution with more general metric Ansatz. The particular case in which both of the harmonic functions $H_{F}$ and $H_{1}$ have two centers corresponds to the "bulk" spacetime counterpart configura- tion to the string junction discussed in the previous section. As a string approaches the D3-brane, it splits into two strings.

By applying the type-IIA-type-IIB $T$-duality transformations along the overall transverse directions of the solution (30), one obtains the following supergravity solution for the fundamental string and the $\mathrm{D} p$-brane ending on the $\mathrm{D}(p$ $+2)$-brane:

$$
\begin{aligned}
& G_{M N}^{\mathrm{str}} d x^{M} d x^{N}=-H_{F}^{-1} H_{p}^{-1 / 2} H_{p+2}^{-1 / 2} d t^{2}+H_{p}^{-1 / 2} H_{p+2}^{-1 / 2} \\
& \times\left(d w_{1}^{2}+\cdots+d w_{p-1}^{2}\right)+H_{p}^{1 / 2} H_{p+2}^{-1 / 2} \\
& \times\left(d x_{1}^{2}+d x_{2}^{2}+d x_{3}^{2}\right)+H_{p}^{-1 / 2} H_{p+2}^{1 / 2} d u^{2} \\
&+H_{F}^{-1} H_{p}^{1 / 2} H_{p+2}^{1 / 2} d y^{2}+H_{p}^{1 / 2} H_{p+2}^{1 / 2} \\
& \times\left(d z_{1}^{2}+\cdots+d z_{5-p}^{2}\right), \\
& e^{\phi}= H_{F}^{-1 / 2} H_{p}^{(3-p) / 4} H_{p+2}^{(1-p) / 4}, \\
& B_{t y}^{(1)}= H_{F}^{-1}, \quad A_{t w_{1} \cdots w_{p-1} u}=H_{p}^{-1}, \\
& A_{t w_{1} \cdots w_{p-1} x_{1} x_{2} x_{3}}=H_{p+2}^{-1} .
\end{aligned}
$$

In the case of $p=2$ (the fundamental string and the D2brane ending on the D4-brane), the harmonic functions are given by

$$
\begin{aligned}
& H_{F}=1+\sum_{i} \frac{Q_{F i}}{\left[\left|\vec{x}-\vec{x}_{F 1 i}\right|^{2}+4 Q_{4}\left|\vec{z}-\vec{z}_{0}\right|\right]^{3 / 2}}, \\
& H_{2}=1+\sum_{i} \frac{Q_{2 i}}{\left[\left|\vec{x}-\vec{x}_{D 2 i}\right|^{2}+4 Q_{4}\left|\vec{z}-\vec{z}_{0}\right|\right]^{3 / 2}}, \\
& H_{4}=\frac{Q_{4}}{\left|\vec{z}-\vec{z}_{0}\right|} .
\end{aligned}
$$

From this supergravity solution, one infers that there should exist the 1/4 BPS threshold bound state of an electric bion and a magnetic bion under the world volume $U(1)$ gauge field of the 4-dimensional DBI theory. This solution originates from the two intersecting self-dual strings on the M5brane world volume [39] through the dimensional reduction along the longitudinal direction of one of the self-dual strings. The corresponding "bulk" spacetime counterpart configuration is

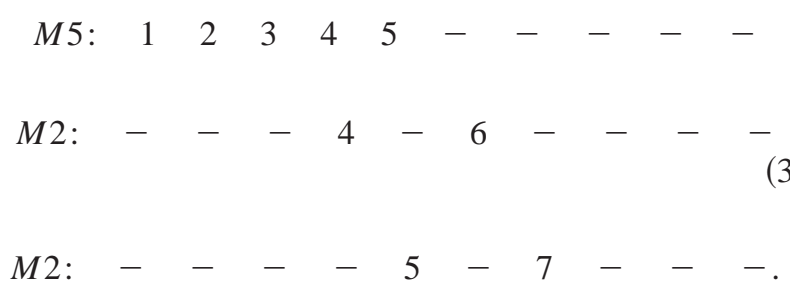


The two world volume self-dual strings are, respectively, stretched along the 4 and 5 directions of the M5-brane world volume. After compactifying either 4 or 5 on a circle, one obtains the above ten-dimensional configuration describing the fundamental string and the D2-brane ending on the D4brane. The corresponding world volume configuration is the threshold bound state of the (electric) 0-brane and the (magnetic) 1-brane in the D4-brane DBI theory. The supergravity solution for the fundamental string and the $\mathrm{D} p$-brane ending on the $\mathrm{D}(p+2)$-brane with $p>2$, which is related to the configuration (37) through the dimensional reduction and the type-IIA-type-IIB $T$-dualities, also implies the existence of the 1/4 BPS threshold bound state of an electric bion (0brane) and a magnetic bion $[(p-1)$-brane $]$ under the world volume $U(1)$ gauge field of the $(p+3)$-dimensional DBI theory of the $\mathrm{D}(p+2)$-brane.
[1] M. Born and L. Infeld, Proc. R. Soc. London 144, 425 (1935); 147, 522 (1934); 150, 141 (1936).

[2] M. Born, Ann. Inst. Henri Poincare 7, 155 (1939).

[3] C.G. Callan, C. Lovelace, C.R. Nappi, and S.A. Yost, Nucl. Phys. B288, 525 (1987).

[4] J. Dai, R.G. Leigh, and J. Polchinski, Mod. Phys. Lett. A 4, 2073 (1989).

[5] R.G. Leigh, Mod. Phys. Lett. A 4, 2767 (1989).

[6] G.W. Gibbons, Nucl. Phys. B514, 603 (1998).

[7] C.G. Callan and J.M. Maldacena, Nucl. Phys. B513, 198 (1998).

[8] P.S. Howe, N.D. Lambert, and P.C. West, Nucl. Phys. B515, 203 (1998).

[9] M.J. Duff, Class. Quantum Grav. 5, 189 (1988).

[10] G. 't Hooft, gr-qc/9310026.

[11] G.W. Gibbons and P.K. Townsend, Phys. Rev. Lett. 71, 3754 (1993).

[12] L. Susskind, Phys. Rev. D 49, 6606 (1994).

[13] M.J. Duff, G.W. Gibbons, and P.K. Townsend, Phys. Lett. B 332, 321 (1994).

[14] L. Susskind, J. Math. Phys. 36, 6377 (1995).

[15] J.M. Maldacena, Adv. Theor. Math. Phys. 2, 231 (1998).

[16] N. Itzhaki, J.M. Maldacena, J. Sonnenschein, and S. Yankielowicz, Phys. Rev. D 58, 046004 (1998).

[17] O. Aharony, S.S. Gubser, J. Maldacena, H. Ooguri, and Y. Oz, hep-th/9905111.

[18] G. Papadopoulos and P.K. Townsend, Phys. Lett. B 380, 273 (1996).

[19] A. Strominger, Phys. Lett. B 383, 44 (1996).

[20] P.K. Townsend, Phys. Lett. B 373, 68 (1996).

[21] P.K. Townsend, Nucl. Phys. B (Proc. Suppl.) 58, 163 (1997).

[22] A.A. Tseytlin, Mod. Phys. Lett. A 11, 689 (1996).

[23] A.A. Tseytlin, Class. Quantum Grav. 14, 2085 (1997).

[24] H. Lu and C.N. Pope, Int. J. Mod. Phys. A 13, 4425 (1998).

[25] J.D. Edelstein, L. Tataru, and R. Tatar, J. High Energy Phys. 06, 003 (1998).

[26] I.Ya. Aref'eva, M.G. Ivanov, O.A. Rytchkov, and I.V. Volovich, Class. Quantum Grav. 15, 2923 (1998).

[27] N. Itzhaki, A.A. Tseytlin, and S. Yankielowicz, Phys. Lett. B 432, 298 (1998).

[28] S. Surya and D. Marolf, Phys. Rev. D 58, 124013 (1998).

[29] C. Chu, P. Ho, and Y. Wu, Nucl. Phys. B541, 179 (1999).

[30] I.I. Kogan and G. Luzon, Nucl. Phys. B539, 121 (1999).

[31] M. Bianchi, M. Green, S. Kovacs, and G. Rossi, J. High Energy Phys. 08, 013 (1998).

[32] C. Park and S. Sin, Phys. Lett. B 444, 156 (1998).

[33] H. Yang, "Localized intersecting brane solutions of $D=11$ supergravity,', hep-th/9902128.

[34] D. Youm, "Partially localized intersecting BPS branes," hep-th/9902208.

[35] A. Fayyazuddin and D.J. Smith, J. High Energy Phys. 04, 030 (1999).

[36] A. Gomberoff, D. Kastor, D. Marolf, and J. Traschen, "Fully localized brane intersections-the plot thickens,", hep-th/9905094.

[37] D. Marolf and A. Peet, Phys. Rev. D 60, 1050XX (1999).

[38] P.S. Howe, N.D. Lambert, and P.C. West, Phys. Lett. B 419, 79 (1998).

[39] J.P. Gauntlett, N.D. Lambert, and P.C. West, "'Supersymmetric five-brane solitons,', hep-th/9811024.

[40] M.R. Douglas, “'Branes within branes,'” hep-th/9512077.

[41] E. Bergshoeff, J. Gomis, and P.K. Townsend, " $M$-brane intersections from world volume superalgebras,' Phys. Lett. B 421, 109 (1998).

[42] J.P. Gauntlett, J. Gomis, and P.K. Townsend, J. High Energy Phys. 01, 003 (1998).

[43] G. Papadopoulos, Phys. Lett. B 434, 277 (1998).

[44] G.W. Gibbons and D.A. Rasheed, Nucl. Phys. B454, 185 (1995).

[45] J.H. Schwarz, Phys. Lett. B 360, 13 (1995); 364, 252 (1995).

[46] G.W. Gibbons and D.A. Rasheed, Phys. Lett. B 365, 46 (1996).

[47] A.A. Tseytlin, Nucl. Phys. B469, 51 (1996).

[48] M.B. Green and M. Gutperle, Phys. Lett. B 377, 28 (1996).

[49] P.K. Townsend, Phys. Lett. B 409, 131 (1997).

[50] D. Berman, Phys. Lett. B 409, 153 (1997).

[51] A. Khoudeir and Y. Parra, Phys. Rev. D 58, 025010 (1998).

[52] M. Cederwall and P.K. Townsend, J. High Energy Phys. 09, 003 (1997).

[53] M. Cederwall and A. Westerberg, J. High Energy Phys. 02, 004 (1998).

[54] D. Bak, J. Lee, and H. Min, Phys. Rev. D 59, 045011 (1999).

[55] J. Gutowski and G. Papadopoulos, Nucl. Phys. B551, 650 (1999).

[56] J.P. Gauntlett, C. Koehl, D. Mateos, P.K. Townsend, and M. Zamaklar, Phys. Rev. D 60, 045004 (1999).

[57] J.H. Schwarz, Lectures on Superstring and M Theory Dualities: Given at ICTP Spring School and at TASI Summer School [Nucl. Phys. B (Proc. Suppl.) 55, 1 (1997)].

[58] K. Dasgupta and S. Mukhi, Phys. Lett. B 423, 261 (1998).

[59] A. Sen, J. High Energy Phys. 03, 005 (1998).

[60] E. Bergshoeff, H.J. Boonstra, and T. Ortin, Phys. Rev. D 53, 7206 (1996).

[61] J.H. Schwarz, Nucl. Phys. B226, 269 (1983). 
[62] P.S. Howe and P.C. West, Nucl. Phys. B238, 181 (1984).

[63] D. Zwanzinger, Phys. Rev. 176, 1480 (1968).

[64] D. Zwanzinger, Phys. Rev. 176, 1489 (1968).

[65] J. Schwinger, Phys. Rev. 144, 1093 (1966).

[66] J. Schwinger, Phys. Rev. 173, 1536 (1968).
[67] T.T. Wu and C.N. Yang, Nucl. Phys. B107, 365 (1976).

[68] T.T. Wu and C.N. Yang, Phys. Rev. D 14, 437 (1976).

[69] E. Witten, Phys. Lett. 86B, 283 (1979).

[70] M.S. Costa and G. Papadopoulos, Nucl. Phys. B510, 217 (1998). 